\title{
Focused Daydreaming and Mind-Wandering
}

\author{
Fabian Dorsch
}

Published online: 7 November 2014

(C) Springer Science+Business Media Dordrecht 2014

\begin{abstract}
In this paper, I describe and discuss two mental phenomena which are somewhat neglected in the philosophy of mind: focused daydreaming and mindwandering. My aim is to show that their natures are rather distinct, despite the fact that we tend to classify both as instances of daydreaming. The first difference between the two, I argue, is that, while focused daydreaming is an instance of imaginative mental agency (i.e. mental agency with the purpose to voluntarily produce certain mental representations), mind-wandering is not-though this does not mean that mindwandering cannot involve mental agency at all. This personal-level difference in agency and purposiveness has, furthermore, the consequence that instances of mindwandering do not constitute unified and self-contained segments of the stream of consciousness - in stark contrast to focused daydreams. Besides, the two kinds of mental phenomena differ in whether they possess a narrative structure, and in how we may make sense of the succession of mental episodes involved.
\end{abstract}

There are at least two distinct mental phenomena that we sometimes categorize as instances of 'daydreaming'. On the one hand, there are what are typically called instances of mind-wandering. ${ }^{1}$ When we sit in a boring lecture or look out through the window of a moving train, we often stop attending to what is before our senses and give our mind relatively free reign. That is, we substantially lessen our control over the flow of mental episodes that constitutes our stream of consciousness and instead let ourselves be surprised and entertained by experiences and thoughts that follow each other more or less spontaneously, and which are typically concerned with frequently

\footnotetext{
${ }^{1}$ Discussions of mind-wandering - sometimes under the label of 'daydreaming' — can be found in, for example, Singer and McCraven (1961), Smallwood and Schooler (2006), Zangwill (2006), Sutton (2010), and Metzinger (2013). Although Singer allows for 'positive constructive daydreaming' which furthermore helps us to achieve some of our personal goals, he does not seem to count focused daydreams among the (central) examples of 'positive constructive daydreaming', but rather conceives of the latter in a way which is compatible with my characterisation of mind-wandering. See McMillan et al. (2013) and Regis (2013) for further discussion, especially concerning Singer's seeming identification of 'daydreaming' with mindwandering.
}

F. Dorsch $(\bowtie)$

Department of Philosophy, University of Fribourg, Avenue de 1'Europe 20, 1700 Fribourg, Switzerland e-mail: fabian.dorsch@unifr.ch 
changing subject matters. From recalling some of the things that we did in our last holidays, we may switch to visualizing how it would be like to sit now at the beach, before thinking about our friend who is in Paris right now, which may then lead us to entertain the possibility of eating out tonight, after which we may imagine how the dinner conversation with our potential date might go, and so on.

On the other hand, we may be engaged in what has been labelled focused daydreaming. ${ }^{2}$ In such cases, we also withdraw from the world surrounding us and start to experience or think about objects and events that are largely absent, past or imaginary. But we take a much more active part in directing the order and content of our mental episodes and usually stick to a particular topic or issue. In preparation of their ride down the track, the drivers of bobsleighs tend to close their eyes and imagine racing the whole run from start to finish, thereby visualizing the alternating curves and straight passages. In fact, someone, who is an ardent spectator of the sport and almost as familiar with the track as the drivers, may engage in more or less the same focused daydream, even though without any intention to follow it up with a real run through the ice canal.

Some psychologists appear to draw the same distinction-for instance, Giambra (1995) who differentiates between 'spontaneous mind-wandering' (i.e. mindwandering in my sense) and 'deliberate mind-wandering' (i.e. focused daydreaming). Schooler et al. (2011, p. 319), on the other hand, seem to intend to encompass both mind-wandering (in my sense) and focused daydreaming when they define 'mind wandering' as 'engaging in cognitions unrelated to the current demands of the external environment'. But some of their writings also suggest that what they have in mind when talking about 'mind wandering' is rather something more passive, namely 'a shift of attention away from a primary task toward internal information, such as memories' (Smallwood and Schooler 2006, p. 946). Accordingly, it is not absolutely clear whether they take focused daydreams to be (central) instances of 'mind wandering'. This illustrates that the terminology in this area is not (yet) fully established and uniform, and that 'daydreaming' and 'mind-wandering' are used in different ways. But I hope that the examples and conceptual considerations given in this paper suffice to clearly individuate and differentiate the two kinds of mental phenomena that I have in mind when distinguishing between mind-wandering and focused daydreaming.

Neither of the two mental phenomena is often discussed in the philosophy of mind. In particular, they are rarely contrasted with each other. In this paper, I aim to show that their natures are indeed quite distinct, despite the fact that we tend to treat both as instances of 'daydreaming'. The first difference between the two, I argue, is that, while focused daydreaming is an instance of imaginative mental agency (i.e. voluntary mental agency with the purpose to produce certain mental representations), mind-wandering is not - though this does not mean that mind-wandering cannot involve mental agency at all. This personal-level difference in agency and purposiveness has, furthermore, the consequence that instances of mind-wandering do not constitute unified and selfcontained segments of the stream of consciousness-in stark contrast to focused daydreams. Besides, the two kinds of mental phenomena differ in whether they possess

\footnotetext{
${ }^{2}$ For example, when McGinn (2004, ch. 6) and Ichikawa (2009) talk about 'daydreaming', what they have in mind is focused daydreaming. See also Metzinger (2013) for discussion.
} 
a narrative structure, and in how we may make sense of the succession of mental episodes involved. ${ }^{3}$

The paper is divided into two large parts. In the first, I describe some of the foremost features of focused daydreams, notably their purposiveness and their narrative structure. Given that they are imaginative mental projects, I concentrate in particular on the nature of mental projects (i.e. complex mental actions). The second part is concerned with mind-wandering and how it differs from focused daydreaming. At the heart of the discussion is the central role of association in mind-wandering and the resulting lack of an overall representational purposes, which prevents instances of mind-wandering from being distinct and unified mental phenomena (such as mental projects), and also from having a narrative structure.

\section{Focused Daydreaming}

In this section, I aim to illustrate that focused daydreams are imaginative mental projects - that is, instances of imaginative mental agency — which, moreover, possess a narrative structure. In order to do so, I first have to say a bit more about the nature of mental projects, before specifically addressing the issue of what it means for them to count as purposive and mental and, in certain cases, also as imaginative. I conclude the section with a description of the narrative structure of focused daydreams, before then moving on in the next section to how focused daydreaming differs from mind-wandering.

\subsection{Mental Projects vs. Mental Episodes}

What I have in mind when speaking of mental projects is perhaps best illustrated by a list of examples: mentally calculating a sum; drawing an inference; making up our minds about what to think or do by considering and weighing in our minds the relevant reasons already available to us; trying to empathize with another person and to predict her thoughts and feelings on the basis of our knowledge about her; developing in our minds solutions to some problem; daydreaming about climbing Mount Everest; reconstructing in our memory the events and conversations of some day in the past; recreating or composing in our minds stories, pictures or melodies; reflecting on our current situation and our various responses towards it in order to get clear about our own feelings; calming ourselves down by meditating or reminding ourselves of something pleasant; imagining the sensory experiences involved in playing a Bach

\footnotetext{
${ }^{3}$ Interestingly, a similar subdivision seems to be present in dreaming. While some sequences of dream episodes are structured and unified by means of their shared link to purposive mental agency (i.e. examples of lucid dreaming), other sequences lack this purposiveness, structure and unity (i.e. 'standard' examples of dreaming). Note, however, that terms like 'dreaming' or 'dream(s)' do not denote a certain class of mental phenomena, but rather a specific state of consciousness (e.g., to be contrasted with being awake, or with being comatose). Furthermore, there are good reasons to assume that there is a fundamental difference between waking consciousness and dream consciousness, which has the consequence that, say, focused daydreaming and lucid dreaming could not belong to the same mental kind. In particular, we should not take (lucid) dreaming to be an instance of imagining. I discuss and defend these claims in more detail in Dorsch (2015).
} 
prelude on the piano; and so on (further examples can be found in Wollheim 1973 and 1984, ch. 3; Peacocke 1985; Budd 1989, ch. 5; and Zangwill 2006).

Mental projects like these are composite mental episodes, that is, complex segments of the stream of consciousness. They are composed of simple(r) mental episodes, such as episodes of perceiving, remembering, imagining, thinking, feeling, desiring or deciding. It is not always easy to say which mental episodes count as composite, and why. But one of the main criterion should probably be whether a given segment of the stream of consciousness involves elements that could occur on their own, that is, independently of being part of the segment. Accordingly, if a mental episode does not allow for such further division, it should not count as a mental project. Like all other mental episodes, mental projects are conscious and episodic phenomena. We have access from the inside to what they are like (or to what it is like for us to have them); and they have a precise duration, with specific starting and end points. It makes sense, for instance, to ask when someone started to calculate a certain sum in its head, and how long it took him. Indeed, their episodic character is central to the unity of mental episodes, on the personal level, as discrete and independent mental phenomena.

However, not all composites of mental episodes are projects - just think of the fairly random sequence of mental episodes that you enjoyed between twelve and one o'clock today. Moreover, two distinct mental projects may occur in our minds roughly at the same time (e.g., immediately following or alternating with each other). For instance, my pursuit of the project of thinking about a particular philosophical problem may temporally overlap with my pursuit of the project of deciding what to write in a letter to a friend; and I may switch my attention from one to the other and back again. This indicates that the differentiation of mental projects from each other and from other complex sequences of mental episodes is a matter of more than just their temporal aspects.

\subsection{Purposiveness and Unity}

The crucial feature in this respect is the personal-level purposiveness of mental projects. It is responsible for the fact that mental projects are single, self-contained phenomena and constitute distinct parts of the stream of consciousness. Without their purposiveness, mental projects could not be distinguished from other phenomena occurring in phenomenal consciousness. In particular, it would be unspecified which simpler mental episodes are constituents of a particular project, and which belong to a different complex mental episode, or to none at all. The purpose of mental projects is what holds their elements together and distinguishes the projects from other mental phenomena, all on the personal level. Indeed, we identify and describe mental projects in terms of their purpose. The projects of empathizing with another person and of deliberating about what to do tonight differ from each other because we engage in them in the pursuit of different ends, namely to better understand the other person and her situation, and to come to a decision with respect to how to spend the evening, respectively.

The purpose of a mental project unifies the latter on the personal level by determining which episodes in one's stream of consciousness are generated or included as part of the project, and which not. What is thereby decisive is whether the generation or inclusion of the episodes contributes (or is taken by us to contribute) to the furtherance 
of the purpose that defines the mental project. ${ }^{4}$ For example, pursuing the project of recalling some past holiday involves reminiscing about certain events that happened during it; and the resulting episodes of remembering become constituents of our project because they contribute to the desired recollection of those holidays. By contrast, the perceptions, thoughts or feelings that we may have at the same time as, but unrelated to, our remembering are not be part of our project because they do not further the purpose of recalling the holiday. Similarly, which episodes are produced or admitted as elements of the project to decide what to do tonight is determined by the end of this project, namely to come to a reasonable practical conclusion. Reminding ourselves of the available options and our evaluations of, or feelings towards, these options are both constituents of our project. Taking into consideration what we currently hear another person saying may be included by us as well, depending on whether it bears on the issue. But feeling an itch or having a spontaneous thought about whether Bovary is really oblivious to the reasons for his wife's behaviour do not enter our project.

What matters for whether a certain (simpler) episode belongs to a given mental project is thus whether the episode helps the project to fulfil its purpose. The precise extent or nature of this contribution may not always be easy to specify. Intelligibility of the link between episode and project seems to be a minimal requirement; which again presupposes some non-causal-or not merely causal — connection between the purpose of the project and the nature or content of the episode in question. Rational links (like instrumental ones) are obviously good candidates. But perhaps other types of connection are relevant as well (e.g., those involved in the narrative structures to be discussed later on). ${ }^{5}$ In any case, without a purpose, mental projects would lack unity and fail to constitute distinct mental phenomena. They would be nothing more than arbitrarily individuated portions of the stream of consciousness. For, apart from purposiveness, no other feature of such segments could unify them and specify which particular mental episodes belong to the project.

The unity could not be due to the obtaining, among the episodes of each project, of certain causal or rational relations. The reason for this is that the respective relations often reach beyond the borders of projects: mental projects do not occur causally or rationally disconnected from other conscious phenomena. When I am engaged in the project of deciding where to go during my summer holidays, a random thought about

\footnotetext{
${ }^{4}$ There is of course the complication that we may be wrong about what contributes to the pursuit of a certain end. We may, for instance, erroneously think that listening to our friend, or relaxing ourselves by thinking of something pleasant, helps us to come to a better decision about what to do tonight. So should our consideration of our friend's opinion, or our mental effort to relax ourselves, count as part of our deliberative project? If they do, we might have to acknowledge that what matters for the inclusion in a given mental project is not real, but only seeming contribution to the achievement of the project's purpose. However, there is no need to settle this issue here; and in what follows, I assume, just for the sake of simplicity, that actual contribution is required. Even if this should turn out to be false, it would still remain true that what unifies mental projects is their purpose.

5 That we do not yet have a full grasp of all the different ways in which mental episodes may contribute to the fulfillment of the purpose of a mental project and thus be part of that project does not imply that there is no definite answer to the question of when - and why - a given mental episode belongs to a particular project. The same applies to the issue of whether mental projects are limited exclusively to the mind (see the following footnote). But even if mental projects would have vague boundaries, this would not deprive them of unity, or of forming a real class of mental phenomena that can be further investigated, empirically or otherwise. Indeed, such investigation is presumably needed to be able to identify more clearly the borders of given mental projects.
} 
my friend may give me the idea to visit Rome - for instance, because I know that it is my friend's favourite city. I may also consider the fact that Rome is located in Italy and can be reached by train in $8 \mathrm{~h}$ - a fact, knowledge of which I acquired by means of past perceptions and judgements. Furthermore, my eventual decision to travel to Rome may give rise to further decisions, such as to check on the prices for train tickets and hotel rooms, or to get into touch with my friends living in Rome. But none of these other mental episodes - the random thought about my friend, the past perceptions or judgements concerning Rome, or the subsequent decisions to further plan the journey-are part of my mental project of deciding where to spend my summer holidays, despite of being causally or rationally related to this project.

Mental projects are also not unified in virtue of something external to them. As it happens, the episodes of a given project need not concern a common object or subject matter. And even if they do, this will simply be another consequence of the particular nature of the purpose of the project in question. When I am daydreaming about my next holidays, the involved mental representations may have many different objects, such as the various friends that I would like to meet, or the various places that I would like to visit. If, on the other hand, all episodes involves are concerned just with Rome and my friends living there, this will be due to the fact that the purpose of my project is more specific, namely to daydream about my next visit to Rome. Since it is difficult to think of further features or factors that might be responsible for the unity of mental projects, it is reasonable to conclude that, in order to constitute distinct mental phenomena in - and not merely arbitrary temporal portions of - the stream of consciousness, mental projects have to be purposive.

The unity of a project does not presuppose that the project is continuously in the foreground of our attention, or even in phenomenal consciousness. When I am interrupted by a telephone call while trying to solve a problem or deliberating about what to do in the summer, I may return to my project and take it up again straight afterwards, or the next day, or the next week. Although my mental project may stay in the background of my mind during the phone conversation and perhaps for some time longer, it need not do so. In particular, I do not have to be consciously occupied with the project during the whole period of the break in order to be able to return to it later on. This is just a reflection of the fact that we do not begin a new project-mental or not - each time we take up again our striving for a particular goal. Indeed, complex projects like writing a book, or trying to understand ourselves or another person, usually have a non-continuous nature. Our engagements with them stretch over months or years and involve numerous breaks, while continuing to be concerned with a single, temporally extended and non-continuous project. In this respect, mental projects are similar to many complex mental experiences, such as aesthetic ones. For instance, we do not begin to read a novel anew each time we take it up again on the page where we left it the last time, but instead typically continue our experience of reading it (and this remains true even when we reread some of its passages in order to remind ourselves, or get a clearer grasp, of them).

\subsection{Purposiveness and Agency}

Apart from being responsible for their identity and unity, the purposiveness of mental projects also contributes to their status as instances of mental agency. That something is 
purposive (on the personal level) means, very roughly, that it is done by us for an end: that is, either just for its own sake, or for an ulterior purpose (Pink 1996, pp. 14f. and 144). We may kick a ball because we simply want to kick it, or because we want to score a goal. In both cases, our kicking of the ball is purposive. We do things for an end on the basis of, and as the effect of, some of our motivational states (or episodes) that present us with the end in question. In our example, what moves us to kick the ball may be our intention to score a goal, say. Similarly, we are engaged in the mental project of calculating a sum in our mind, or of imagining to be in the shoes of someone else, because this is what we have decided to do.

The motivational role of intentions and similar mental states is thereby multifaceted. They initiate and sustain purposive doings; they guide them until their completion or interruption; and they help us and others to make sense of what we are doing. We go through the motions of kicking the ball because, and as long as, we want to score a goal; we try to kick the ball in a way which we believe is most likely to lead to a goal being scored; and that we kick the ball in this way is intelligible in the light of our wish to score a goal. Accordingly, what is distinctive of motivational states is that they possess the - not necessarily always realized - power to initiate, sustain, guide and explain purposive doings. Many different kinds of state (or episode) have been proposed as candidates for motivational states, such as desires, volitions, tryings, urges, impulses, wants, wishes, emotions, evaluative or practical judgements, and so on. Out of convenience, I focus here on intentions as examples of states with motivational power.

The link to motivational states already indicates that purposiveness is closely related to agency. Indeed, some philosophers have argued that all purposive doings - including all engagements in mental projects - count as actions (Pink 1996, pp. 14f. and 42; 2003). But this would mean that there can be involuntary mental actions. For example, deciding to do something is purposive, but not done at will (Pink 1996, ch. 7). When we come up with a particular decision about what to do tonight, say, we do not make this decision simply in response to wanting to make it (i.e. independently of whether we will ever act on it), but instead in response to some other purpose (e.g. because we want to spend a pleasant evening and have some opinions about what constitutes such an evening). Rejecting the possibility of involuntary actions, many philosophers have therefore limited agency to purposive doings that are done at will (e.g. Davidson 1980, ch. 4; see also Pink 2003).

Mental projects turn out to be instances of agency, independently of whether we accept purposiveness or voluntariness as the mark of agency. For our engagement in mental projects is not only purposive, but also voluntary. When we are creating a story in our mind, or try to relax ourselves by means of visualising something pleasant, we do this at will, that is, because we want to do it (even if there is some ulterior purpose that drives our voluntary engagement in the project). However, that mental projects are instances of agency does not require that all their episodic constituents are instances of agency (Wollheim 1973, pp. 70f.). Mental projects normally involve at least some episodes which have been generated by passive mechanisms, such as association, epistemic processes, or the manifestation of mental dispositions. In this respect, mental projects do not differ from other forms of agency. Actively raising one's arm, say, involves the stimulation of certain nerves; while scoring a goal exploits the effects of gravity and also the movements of the other players (which are passive in so far that 
they are beyond the control of the scorer). Relatedly, the unity of mental projects does not presuppose that all (or perhaps even any of) the episodes included in a project come into being during our pursuit of it. Instead, they may arise independently of the project and only afterwards become one of its parts - say, due to some act of active inclusion on our behalf, or simply because they happen to help us in our pursuit of the project.

Some mental projects may possess purposes which render them open-ended in the sense that there is no specific point at which their purpose counts as achieved. Such projects do not finish by themselves, but have to be terminated by some factors external to them - say, an act of will, or some distracting influence. When I am engaged in daydreaming about the likely consequences of climbing Mount Everest on my life and the lives of others, there need not be any clear point within the daydream at which I count, or recognize myself, as having finished my project: not after visualizing myself reaching the summit; nor after returning in my imagination to the base camp, or Katmandu, or home; nor after imagining the reactions of my friends to my bragging about my feat, nor after speculating about the fame and fortunes which will await me and completely change my life if I sell my story. Of course, the more details of my climb I imagine, the richer and more complete my daydream might become. But there is no richest or most complete daydream to be had: I could literally go on and on, even beyond imagining my own death (e.g. when I imagine my posthumous fame).

Similarly, there need not be any clear point at which I begin to be successful in daydreaming about climbing Mount Everest. For there is no easily specifiable lower limit of richness or completeness which separates success and failure. It is unclear when I begin to count as, say, daydreaming about the climb: when I suppose that I am climbing; when I visualize a certain stretch of my climb; or already when I imagine arriving in Katmandu and realizing that my climbing gear got lost on the flight and I have to give up on my ambitions. This does not imply, however, that we cannot fail in pursuing open-ended projects. On the one hand, we can fail to engage in them at all (e.g., if I fail to form any imaginative representation, or if I represent myself solely as lying in the sun on the Riviera). On the other hand, the daydream may not be rich or complete enough (e.g., when I intend to imagine not only my climb, but also its consequences for my life, but terminate my imaginative activity before reaching this second point in my daydream). In all these respects, open-ended mental projects are similar to, say, the non-mental project of going for a walk: the latter also has no clear boundaries, does not by itself impose any stopping point, yet may nonetheless fail.

\subsection{Mental Projects vs. Non-Mental Projects}

Most of what I have said so far about the purposiveness, unity and agency of mental projects also applies to non-mental projects, like building a house, writing a novel, teaching someone to speak a new language, preparing a party or raising a child. But there are also crucial differences between the two kinds of project.

First of all, projects count as mental only if - and because - they aim at altering some facts about one's own mind. In other words, mental projects have the purpose of bringing about (or otherwise influencing) certain kinds of mental phenomena in one's own mind, whether these are episodic or dispositional in character; and they count as accomplished if the respective mental phenomena indeed come into being. Thus, mental projects aim at, say, the acquisition of beliefs or character traits, the 
manifestation of mental dispositions (such as of a mnemonic or emotional nature), the formation of judgements, intentions or imaginative representations, the alteration of one's mood, or perhaps the distraction from, or repression of, certain worries or feelings. In contrast, non-mental projects (such as the ones mentioned) typically aim at bringing about facts which are either non-mental or concern the mind of other persons.

Having the purpose of influencing one's own mind is, however, insufficient to distinguish mental from non-mental projects. Consider the project of improving your mood. You may engage in this project by turning on and listening to a piece of music of which you know that it is likely to cheer you up. But, instead, you may also simply auditorily imagine singing or listening to the song. You may improve your mood in both ways, but only the second method constitutes an engagement in a mental project. Examples like these suggest the addition of a further requirement on mental projects, namely that they occur - from their conception until their completion-exclusively in the mind. Indeed, if a project has the purpose to bring about some mental phenomenon in one's own mind and does not involve any nonmental events or elements, then it is safe to say that it is mental project. ${ }^{6}$

Different kinds of mental project differ in respect of their purposes and, hence, also in respect of the mental episodes that constitute our engagement with them. Cognitive projects, for instance, aim at the acquisition of knowledge; while imaginative projects aim at the voluntary representation of specific states of affairs - or so I argue in (Dorsch 2012, chs. 13f.). This difference is strict, given that we cannot acquire knowledge by forming a mental representation at will. Furthermore, there are different types of imaginative projects, such as empathizing with another person (i.e. imagining having her feelings and thoughts and being in her position in the world), engaging with the fictional world represented by a novel or movie (i.e. imagining what happens in that world and having certain perceptual or emotional experiences with respect to the fictional characters and events concerned), setting up and running a thought experiment (i.e. supposing certain things to be true and investigating their consequences) - or, indeed, focused daydreaming.

Instances of focused daydreaming count as (possibly open-ended) mental projects and, more specifically, as imaginative projects because they possess an overall purpose, which is to voluntarily form representations with specific contents (e.g., contributing to the representation of a climb of Mount Everest). What we are daydreaming about is

\footnotetext{
${ }^{6}$ The requirement that the whole project has to take place within the mind might sometimes be too strong. While most mental projects consist solely of mental episodes (and perhaps also dispositions) and the mental actions and processes which link these together, it may be argued that some mental projects involve also certain forms of relatively non-interfering bodily actions. For instance, it does not seem to matter much for the project of finding the best next move in a given game of chess whether one scans the position on a chess board with one's eyes or instead visualizes it in one's mind. But do the movements of one's eyes render the project in question non-mental? One way of capturing such cases would be to modify the second requirement on mental projects - for instance, by requiring that mental projects are such that they merely could (but need not) be pursued without any involvement of bodily action or other external events. Following the suggestion of one of the referees, another option is to treat cases like this as examples of embodied cognition (see Anderson, 2003, sect. 3.3, for a discussion of similar cases). Treating a project as dependent on some bodily movement in this way might still be compatible with the idea that the project itself is limited to the mind (e.g. when the dependence is understood as being weaker than proper constitution). In any case, the mental projects that interest us the most - focused daydreams - clearly occur exclusively in the mind.
} 
determined by some underlying motivational states which guide our mental agency by moving us to generate or include episodes with relevant contents, and to link them to each other in accordance with the general representational purpose of the activity of daydreaming in question. In other words, the motivational states determine the episodic constituents, course and general content of our focused daydreams. In particular, focused daydreaming often starts off from an assumed premise, generally reflecting the intended content of the activity of daydreaming, and is then actively developed from there step by step, in a way very similar to that of stories.

For instance, my daydream about climbing Mount Everest originates in my intention or desire to form representations that are concerned with, and jointly constitute a representation of, such a climb. Daydreaming with this general purpose in mind may begin with the assumption that I am about to climb Mount Everest, and progress from there by means of the production or incorporation of a series of representations, linked to each other and to the initial assumption in virtue of the overall purpose of representing a climb of Mount Everest. Such representations may concern the preparations for the climb, the actual way up the mountain, the view of the surrounding landscape, the intermingled feelings of exhaustion, cold, fear, excitement and craziness, the distancing thought expressing my knowledge that I could never actually bring myself to climb Mount Everest, the consideration of what might bring people to attempt the climb, the experience of reaching the summit and of the sudden irrelevance of everything (including one's safe return), and so on. All these mental episodes and projects make up the complex activity of daydreaming about how it would be to climb Mount Everest.

The episodes' occurrence, content and relationship to each other are motivated and guided by my intention to daydream about climbing the mountain; and, together, the episodes contribute to the achievement of that purpose.

A full account of focused daydreaming would have to say more about whether - and if, how - they differ from other types of imaginative project. In some cases, this task seems fairly easy. For instance, focused daydreams are not responses to representational artworks and differ in this respect from our from imaginative engagements with the latter. But it is less clear whether there is a real difference between focused daydreaming, on the one hand, and empathy or thoughts experiments, say, on the other. One noticeable difference is perhaps that the last two are always embedded in more encompassing cognitive projects, while the first is often not. For example, we may imagine having the experiences and thoughts of another person because we want to find out how she feels (empathy), or because we just enjoy imagining being her or in her position (daydream). Moreover, such differences in purpose engender differences in the mental episodes that make up the respective imaginative projects. When empathizing, we try to be as accurate as possible with respect to the other person's mental states and thus rely on, and incorporate into our project, our knowledge about her. By contrast, there are no such constraints on daydreaming about being the other person (e.g., we can daydream about being a certain celebrity and seeing the world with her eyes although we might not know much about what is going on in her mind). However, empathizing with another person in order to understand her better-as well as running a certain thought experiment, for that matter - may ultimately be nothing but a focused daydream, applied to some secondary cognitive purpose. This hypothesis receives further support from other examples, like the one mentioned at the beginning. 
Visualizing a ride down the ice canal is also an instance of focused daydreaming that we may engage in simply for the sake of doing so, or alternatively because of some ulterior purposes (e.g., to prepare for a real run).

\subsection{Narrative Structure}

In any case, what is characteristic of focused daydreaming - but perhaps not of all other types of imaginative project - is that it possesses a narrative structure. This aspect of focused daydreaming is central to its distinction from mind-wandering, which is why it is worthwhile to look at it more closely. However, it is admittedly not easy to specify precisely what it means for a sequence of episodes to have a narrative structure. For our purposes, it suffices to highlight the fact that there are five features that are characteristic of many, if not all, sequences of mental representations with a narrative structure (what I call 'narratives').

One of these features is that the entities that the episodes in the sequence are about are frequently represented as behaving in regulated ways which facilitate our understanding of their behaviour. For instance, physical objects follow natural laws and are used in accordance with conventions; while persons think and act in rational or at least comprehensible ways. This enables us, for instance, to identify or empathize with the characters in stories; or to make sense of the causal nexus between certain narrated events (Williams 2002, pp. 233ff).

The regularity of narratives is closely related to another aspect of narrative sequences: that they often portray some development concerning the represented entities. Movement, metamorphosis, maturation, or revolution, are all possible examples of such a development. A narrative may be about the movement of balls on a snooker table, or about the alteration in their colours; it may be about the change in a character's opinions, or about the overthrow of a government.

In addition, both the feature of regularity and that of development presuppose a third aspect: that the relevant episodes in the narrative sequence concern the same particular entities. More than one mental episode is needed to represent a pattern of behaviour or a process of evolution. And the episodes involved have to be about the same thing, namely whatever acts or changes in the way in question.

A fourth important characteristic of many narrative sequences is that they reveal a certain perspective on what is being told - whether this perspective is very subjective and evaluative or emotional, as in the case of, say, many first-personal narrations; or more objective and distanced, as in the case of, say, classical instances of an omniscient narrator (Goldie 2003a, b).

A last characteristic of narratives is that they usually portray the events they represent as temporally ordered: one thing is represented as happening either simultaneous to or after another (Carroll 2001, p. 120; Lamarque 2004, p. 394). There are presumably further features distinctive of many, if not all, narratives. But noting these five characteristics should suffice to clarify the difference between focused daydreaming and mind-wandering with respect to their intelligibility.

Focused daydreams typically possess several or all of the features just described. For instance, many of the episodes making up my daydream about climbing Mount Everest 
concern the same individual entities (such as people, tools, peaks, etc.), which are thus often characterized by means of more than one episode. Then, the daydream is likely to represent objects as behaving in regulated ways (e.g., avalanches or ice-axes obeying gravity; or despairing and panicking climbers following their temperament or general human nature). Furthermore, the daydream portrays many kinds of development (e.g., the changes in daytime, weather, height, feelings, and so on). And it normally does this by means of arraying certain events in time (e.g., the ascent as happening before the fall). Finally, the daydream as a whole — or some considerable part of it - may disclose some of my own feelings or opinions about attempting to climb Mount Everest (e.g., my fear and fascination, or my assessment that many people taking up this challenge do not really know what they are doing).

\section{Mind-Wandering}

The other classical example of what we sometimes take to be daydreaming is mindwandering. Just like focused daydreaming, mind-wandering involves sequences of connected mental episodes. But, this time, the connection is not —or not primarilydue to imaginative purposiveness and mental agency, but instead to association and similar causal factors. After saying a bit more about two different kinds of associative mind-wandering and their difference in temporal extension, I highlight two central aspects of focused daydreams that instances of mind-wandering lack: representational purposiveness, and unity. In addition, the two phenomena also differ typically in whether they possess a narrative structure, and strictly in how the succession of mental episodes involved is intelligible to us. We therefore should conclude that, while focused daydreams are imaginative mental projects, instances of mind-wandering do not even constitute distinct and unified sequences of mental episodes with clear temporal boundaries.

\subsection{Two Kinds of Associative Chains}

Mind-wandering occurs when reduce our own mental activity, let our mind 'wander off freely' and switch our attention from our actual surroundings to the sequence of experiences and thoughts unfolding in our minds. We are especially prone to mindwandering if we are in a relaxed mood and do not have any urgent business to take care of-for instance, when we are taking a bath, lying in bed and waiting for sleep, meditating, sitting in a boring lecture, or looking outside through the window of a moving train.

The most basic forms of mind-wandering consist in sequences of simple, associatively linked mental episodes. What happens in such cases is that we abstain from any active intervention and, so to speak, 'lean back' and 'watch' the series of experiences and thoughts that enter our stream of consciousness due to associative and similar passive forces. Indeed, very different causal factors or processes may be responsible for the occurrence of the mental episodes concerned, and thus for moving forward the instance of mind-wandering that is constituted by those episodes. But what they all have in common is that they are passive: they bring about each of the episodes without the help of our agency (even though we may actively trigger their initial coming into 
operation). ${ }^{7}$ Furthermore, they often (though not always) give rise to new mental episodes in virtue of the representational contents of the old ones (an aspect of mindwandering to which I return shortly). In what follows, I therefore use the term 'association' to refer collectively to the various passive, and often content-related, factors responsible for the production of mental episodes in mind-wandering. Here is how James describes an example of the kind of associative chain that I have in mind:

[...] our musings pursue an erratic course, swerving continually into some new direction traced by the shifting play of interest as it ever falls on some partial item in each complex representation that is evoked. Thus it so often comes about that we find ourselves thinking at two nearly adjacent moments of things separated by the whole diameter of space and time. Not till we carefully recall each step of our cogitation do we see how naturally we came [...] to pass from one to the other. Thus, for instance, after looking at my clock just now (1879), I found myself thinking of a recent resolution in the Senate about our legal-tender notes. The clock had called up the image of the man who had repaired its gong. He had suggested the jeweler's shop where I had last seen him; that shop, some shirtstuds which I had bought there; they, the value of gold and its recent decline; the latter, the equal value of greenbacks, and this, naturally, the question of how long they were to last, and of the Bayard proposition. [...] Every reader who will arrest himself at any moment and say 'How came I to be thinking of just this?' will be sure to trace a train of representations linked together by lines of contiguity and points of interest inextricably combined. This is the ordinary process of the association of ideas as it spontaneously goes on in average minds. (James 1890, pp. 539f.)

And James continues to present another example taken from Hobbes:

In a Discourse of our present civill warre, what could seem more impertinent, than to ask (as one did) what was the value of a Roman Penny? Yet the Cohærence to me was manifest enough. For the Thought of the warre, introduced the Thought of the delivering up the King to his Enemies; The Thought of that, brought in the Thought of the delivering up of Christ; and that again the Thought of the 30 pence, which was the price of that treason: and thence easily followed that malicious question; and all this in a moment of time; for Thought is quick. (Hobbes 1651/1996, part 1, ch. 3, init.)

As Hobbes rightly notes, such associative sequences of simple mental episodes (i.e. episodes of seeing, feeling, thinking, and so on) are usually not very long. Indeed, both examples illustrate well that we often do not notice all the associative steps during their occurrence and have to retrospectively reconstruct-or, in James' words, 'carefully recall'- these intermediary steps after the experiences and thoughts concerned have

\footnotetext{
${ }^{7}$ See Smallwood (2013) for discussion, who also distinguishes between the initial causes of instances of mind-wandering (which may be voluntary or involuntary) and the mechanisms that are responsible for the development and continuity of the sequence of mental episodes in question.
} 
already disappeared again (Zangwill 2006, p. 265). Now, there are at least three reasons for the relative shortness of sequences of associatively linked simple mental episodes.

First, mental episodes do not remain in existence for longer periods of time, unless they are actively sustained by us and thus incorporated into some mental project (e.g., a focused daydream), or caused by underlying factors that stay efficacious for longer stretches (e.g., hallucinogenic drugs or psychological disorders; see Dorsch 2010, p. 187, and 2012, ch. 14.4). Association is normally not among these factors, not the least because the original episodes that associatively give rise to the new ones are often themselves rather short-lived.

Second, many mental episodes occurring as part of associative chains do not themselves lead to further episodes by means of association - either because there are no corresponding associative links or dispositions established in our minds, or because we simply start to become mentally occupied with other things. As a result, many associative sequences of experiences and thoughts come to a halt or peter out after a short while.

Third, it is rather difficult for us to remain purely passive for very long with respect to our mental lives. That is, it is not easy for us not to give in to our tendency to switch attention from one thing to another, and to try to take control of the sequences of nonperceptual experiences and thoughts in our mind. This restlessness of our mind and will is reflected, for instance, in the difficulty of, and the many failed attempts at, meditation.

But there are other instances of mind-wandering that last much longer-most prominently, sequences of associatively linked focused daydreams (and possibly other kinds of mental project). We often engage in a series of successive focused daydreams. And the switches from one to the next are frequently facilitated by the spontaneous occurrence of mental episodes in virtue of some associative links (O'Shaughnessy 2000 , p. 216), or indeed by what James in the passage quoted earlier calls 'the shifting play of interest' (e.g., when some new object, event or topic catches our attention). What we then get are temporally extended instances of mind-wandering. What happens in such cases is that some episodic constituent of the current daydream (or perhaps also an unrelated, but contemporaneous episode) associatively brings about another mental episode which subsequently figures as the starting point for a new daydream, or at least instils in us the manifest desire or intention to begin a new daydream.

Such a sequence of associatively linked focused daydreams may look like the following: I am daydreaming about walking the streets of London and, due to some memory of or fondness for British barber poles with their red and white stripes, this includes visualizing such a pole at the entrance to a barber shop; the colour and name of this pole give rise, by means of association, to the image of the Polish flag and hence to some thought about Poland; because of some long-standing desire to visit that country, I stop my daydream about London and begin to occupy myself instead with a daydream about visiting Poland, starting off with the thought of this country; this again may remind me of my Polish friend at school whom I have not heard of for years, since she entered art school; and I may thus begin to daydream about her subsequent life and about meeting her again.

Indeed, such associative sequences of focused daydreams (and, possibly, other mental projects) are more typical examples of mind-wandering than the shorter associative chains of simple episodes. When we are lying in the bathtub, say, we tend to let 
our mind wander freely for more than just brief moments. This requires that the respective instances of mind-wandering are not simply a matter of association, but involve us as agents. For, again, it would be almost impossible to not actively intervene with sequences of spontaneously occurring experiences and thoughts, or for such sequences to continue without any active sustainment, for any prolonged period of time. Accordingly, when letting our mind wander off, we typically alternate between passively witnessing the spontaneous occurrence of experiences and thoughts and actively developing some of these experiences and thoughts into more expansive daydreams. When one of our a focused daydreams terminates, we often return to a short period of mental inactivity before moving on to the active pursuit of a new daydream. In other words, mind-wandering is often a mixture of association and focused daydreaming (or, more generally, mental projects).

Our occupation with such associative sequences of focused daydreams is very similar to the activity of more or less aimlessly wandering around a city. ${ }^{8}$ During the walk, most - if not all — of our steps or turns are made intentionally. But they are usually not governed by an overall purpose directing them towards a certain destination, say, or giving them an intelligible pattern (e.g., when we aim to turn right at every second corner). For where we direct our steps to is often determined by factors beyond us: because something catches our eyes; because we just follow other people; because there occurs an unexpected opening between the houses or walls; and so on. Similarly, when we let focused daydream follow focused daydream, we are in control of the particular daydreams, especially of their inception. But our various daydreams typically do not share an overall representational goal: they normally do not concern the same subject matter or even the same characters, places, events, and so on. Accordingly, where we finally end up in either case is not determined by our motivational states, in particular not by our desire or intention to wander off freely on the streets or in our mind. Instead, various factors, such as associations, whims, distractions, external stimuli, and so on, contribute to our activity of walking or daydreaming by suggesting possible new directions to be pursued.

Now, mind-wandering - independently of whether it involves associative chains of simple mental episodes or more complex mental projects-differs from focused daydreaming in several important respects. Most prominent among them are four closely related differences, of which the first is the most fundamental in the sense that it helps to explain the presence of the others. The four differences concern, respectively, the representational purposiveness, the narrative structure, the intelligibility and the unity of the segments of the stream of consciousness in question. While the second difference may be just typical or gradual (i.e. may hold normally, but not always), the other three are strict differences that separate the two mental phenomena in a clear-cut manner, rather than locating them on a continuum.

\subsection{The Difference in Representational Purposiveness}

Focused daydreaming and mind-wandering do not generally differ with respect to whether we can engage in them purposively or even voluntarily. We can decide to let

\footnotetext{
${ }_{8}^{8}$ Purely associative sequences would be more like sitting on a bus and letting it take us wherever it goes. We remain completely passive and just watch the changing scenery.
} 
our mind wander off freely in order to achieve a certain goal, in the same way in which we can decide to lie down and stop moving in order to achieve a certain end (e.g., to rest our mind or body, or to enjoy the pleasure of not actively doing anything). ${ }^{9}$ But, in contrast to focused daydreams, instances of mind-wandering do not possess a representational purpose, that is, a purpose which puts some constraints on what the mental episodes aimed for are meant to represent.

Consider, again, the two examples that Hobbes and James describe. Which episodes are included in the respective chains of simple mental episodes is not a matter of some underlying intention to form or have mental representations with a certain content. Rather, what is operative and responsible for the constituents and progress of the sequence is the force of association. Accordingly, the two associative chains lack any representational purpose and, specifically, any imaginative purpose. They are not unified by a common representational aim.

The same is true of associative sequences of focused daydreams. Although each instance of focused daydreaming is determined by imaginative agency, the resulting associative chain is not unified by an overall imaginative purpose and therefore does not show any continuity in what is represented. The switch from one focused daydream to another is instead accompanied by a switch of imaginative purpose, as well as by a switch in the represented entities. In other words, the associative sequence of focused daydreams is non-purposive in so far as it lacks an overall representational purpose; but it is purposive in so far as it consists in a series of particular focused daydreams, each of which is purposive. Sequences of associatively linked focused daydreams differ thus both from single focused daydreams (because of the lack of an overall purpose) and from associative sequences of simpler, nonpurposive mental episodes (because of the involvement of mental agency).

Elsewhere, I have argued at length that imagining is essentially an action, in the same sense in which walking or jumping are essentially actions (Author 2012, chs. 13f.). More specifically, I have identified imagining with the mental action of exerting voluntary control over which specific states of affairs our respective mental episodes are representing. In other words, when we are imagining something, it is at least to some considerable extent up to us which objects we are imagining as having which properties (e.g., whether we are imagining something about a unicorn, say, as well as which size or colour we imagine the unicorn as having). If this view is right, the difference in representational purposiveness is accompanied by a difference in imaginativeness: while focused daydreaming is an instance of imagining, mind-wandering is not. Of course, some or even all of the individual mental episodes associatively linked in mind-wandering may still count as imaginative. But their overall association cannot because it lacks the required representational purpose.

\footnotetext{
${ }^{9}$ See Giambra (1995), Smallwood and Schooler (2006) and Smallwood (2013). Wegner (1997) claims that mental agency may also give rise to mind-wandering in another way. His view is that, often when we introspectively check whether our deliberate attempt at focusing our mind on something particular has been successful, we cause our mind to wander off precisely because of our active intervention to the contrary. Independently of its merits, this proposal is compatible with the view defended here that, although mindwandering is not an instance of imaginative agency, we may actively engage in it by choosing to let our mind wander off. The kind of impact of agency that Wegner identifies is much more indirect and, since it treats mind-wandering merely as an unintended causal effect of mental agency, also neither intentional, nor open to introspection.
} 


\subsection{Some Similarities in Intelligibility}

Because of their fundamental difference in representational purposiveness, focused daydreaming and mind-wandering also differ in three other respects, all of which concern the nature of the connections between the mental episodes involved. Before addressing these further differences, it is worthwhile to point out some similarities in the intelligibility of the connections between the episodic elements.

Both in focused daydreaming and in mind-wandering, the links between the mental episodes concerned are in principle intelligible: we usually can account for the occurrence of the episodes involved by making sense of the connections in play (see Goldie's notion of the coherence in narratives in his 2003a and b). For instance, that I am visualizing a landscape of snow-covered mountains or imagining the feeling of elation and exhaustion when reaching the summit of an eight-thousander can be explained by reference to my intention to daydream about climbing Mount Everest: the latter is what motivates the occurrence of the former. Similarly, Hobbes' thought of treason can be traced back to his occupation with the war between the Royalists and the Parliamentarians, raging in his times, which he knows to involve the handing over of the king to the English Parliament: thinking about the war has caused Hobbes to think about treason because the two representations concern events which are, for him, significantly linked (i.e., the deliverance of the king and the war in question).

Moreover, the rationale of both focused daydreams and associative chains is intelligible on the basis of reflecting on the introspectible features of the mental episodes involved, notably their contents. My underlying intention explains my engagement in the imaginative activities because the contents of the episodes satisfy the description (which is part of the content of the intention) of what I have been meaning to represent. This match makes it comprehensible why the intention has given rise to the specific representations in question, and not to others. Similarly, Hobbes' thought about the civil war accounts for the occurrence of his thought about treason because there is for him a recognizable link between the two represented events (e.g., because he knows the two represented events to be connected through the figure of the King). It is hence legitimate for him, as well as us, to judge that the two thoughts are coupled in virtue of some associative link.

As a result, both focused daydreams and sequences of associated episodic representations are intelligible in virtue of the contents of the mental states in question (and perhaps also some of their other introspectible features, such as their affective character). Furthermore, both differ in this respect from sequences of mental episodes which are merely temporally or causally related to each other and succeed each other for reasons other than what they represent.

\subsection{The Difference in Narrative Structure}

However, focused daydreaming and association differ in the nature of their intelligible structure. The difference can perhaps be best expressed by saying that, at least typically, only focused daydreams are narrative, or can be narrated. I have already argued that focused daydreams show many, if not all of the five features that are characteristic of narrative structures, namely the representation of regularities, developments and temporal order, the continuous reference to the same entities, and the manifestation of a 
specific perspective on what is represented. In contrast, the sequences of associatively linked episodes involved in mind-wandering normally do not show any of the five features, at least not to a significant extent.

In both James' and Hobbes' examples, the mental representations involved do not make sense of regularities among entities. The represented people (e.g., the watchmaker, or the king) are not really represented as thinking or acting in certain ways and hence are not subject to our understanding. The same is true of the represented objects: the conventions and economic principles governing the flotation and exchange of currencies or similar values remain undisclosed (although their existence and influence may be conveyed); and it is not clarified how the war unfolded, or why the clock had broken down. There are some traces to be found of the rationality of subjects or of the behaviour of physical entities: the deliverance of the king is represented as treason and as motivated by greed; and it is suggested that the clock is working again after having been repaired. But the respective forms of behaviour are not fully or richly represented: they are only mentioned or hinted at.

Moreover, the forms of behaviour (as well as most other entities) are typically represented by single episodes, with the result that grasping their presence does not require grasping any associative connections. That treason is motivated by greed is part of the content of a single thought: it is merely stated and not further elucidated. This explains why mind-wandering usually does not reveal or permit the same kind of meaningfulness and understanding of regularities as daydreaming. The relative isolation of associatively connected episodes is also closely related to the fact that such sequences normally do not portray any significant developments: the episodes do not link up to represent changes, or the influence of dynamic forces, over time - as, again, illustrated by James' and Hobbes' examples quoted above. It is said that the value of gold has declined; and the idea of war also involves the notion of some development. But anew, both representations are restricted to single episodes (or even concepts) and do not provide any rich or informative grasp of the stated developments.

That any traces of a representation of regularity or development are typically limited to single thoughts or images is further explained by the fact that not many of the entities represented by members of associative sequences are referred to by more than one episode - and, in the examples, none by more than two. That both the image of the clock and the thought about the man who repaired it make reference to the clock does not establish any narrative link between the two representations - and, in particular, no development or law-like connection. The same applies to the two thoughts about the occurrence of treason: they have nothing in common and are not further linked, over and above being thoughts of the same person and sharing the same subject matter or object (in a wider sense).

Finally, the associative sequences in both examples of mind-wandering do not manifest any perspectives or attitudes in addition to those expressed by each of the single episodes. Hobbes' thoughts assess the deliverance of the king to be treason and do not fully approve of asking the question about the value of a Roman penny. But they do so not in virtue of their associative or other links, but simply by being the thoughts that they are. They would reveal the same view on the events in question if they occurred on their own. Accordingly, the associative links are not essential to the disclosure of Hobbes' assessment. 
What these considerations indicate is that sequences of associatively linked episodes typically do not possess a narrative structure. As the particular nature of the quotes of James and Hobbes indicate, any attempt at narrating what is going on in our minds when we let them wander freely produces just a list of successive and associated mental representations, but not anything like a cohesive a story (Zangwill 2006, p. 264). Of course, mind-wandering may still involve narrative elements, namely if the associatively linked episodes are focused daydreams, each with their own narrative structure. But even then, the resulting instance of mind-wandering as a whole still lacks an overall narrative structure because of the non-narrative associative transitions from one focused daydream to another.

The main reason for this is, of course, the lack of any representational purpose. Focused daydreams possess a narrative structure exactly because they are unified by the purpose of representing a particular scenario that involves temporally ordered events and objects behaving in regular and progressive ways. That is, it is precisely because focused daydreams are guided by the intention to form representations with a specific content that the episodic constituents of the daydreams end up concerning the same entities and relationships, as well as establishing a coherent point of view on the latter. Associative links, by contrast, normally do not engender the same kind of narrative cohesion because of their disconnection from any representational unity.

\subsection{The Difference in Intelligibility}

The difference in narrative structure between focused daydreaming and mindwandering is closely related to another difference that pertains to how we make sense of particular instances of each of the two mental phenomena. In the case of a focused daydream, the connections between the episodes concerned make sense to us in the light of the general purpose or subject matter of the daydream primarily because of the links between the entities that the episodes represent (i.e., the relationships, regularities and developments among those entities). We trace the coherence of the daydream back to the causal or other connections between the represented entities. In other words, what matters and is intelligible for us is how the portrayed entities are linked to each other. This is part of the explanation why daydreams are narratable. But for the intelligibility of daydreams, it is not necessary that their episodes themselves are causally linked to each other, or that we become aware of any causal links that may obtain among them. The experiences and objects imagined during my daydream are unified by the narrative way in which they contribute to the imaginative purpose of my daydream (i.e. by giving rise to the mental representation of a potential climb of Mount Everest). This is sufficient to provide the daydream in question with coherence. The representations involved need not - and often do not — causally influence each other.

In the case of an instance of mind-wandering, on the other hand, coming to understand the connections between the episodes involved means coming to understand their specifically associative causal links. And this requires taking into account their contents (or their other features open to introspection) only in so far as they are causally or otherwise linked to each other in virtue of what they represent. In fact, two kinds of representation may be associated in our minds for many different reasons, not all of which pertain to their contents: because they represent the same, or similar, objects (e.g., a particular clock); or instead the same features of objects (e.g., being an 
instance of treason); or because we once experienced the represented entities at roughly the same time or location (e.g., seeing the watchmaker in the jewelry shop); or while being in a comparable mood (e.g., when realizing that one is in love); and so on. But how the represented entities are causally or otherwise related to each other is typically irrelevant. Instead, what counts and is intelligible is primarily the specific causal or similar links between the associated mental episodes.

Hence, mind-wandering differs in its intelligible nature from focused daydreaming. With respect to focused daydreams, we make sense of the relationships among the entities which the mental episodes portray; while with respect to sequences of associatively linked mental episodes, we make sense of the relationships among those episodes themselves. Again, this difference can be traced back to the more basic difference in what links the mental episodes in the respective segments of the stream of consciousness. If the connections are due to some shared representational purpose, they primarily concern what is represented; while if the connections are due to association, they pertain to the associated episodes themselves.

\subsection{The Difference in Unity}

The last difference between instances of focused daydreaming and instances of mindwandering consists in the fact that only the former, but not the latter, constitute distinct and self-contained parts of the stream of consciousness, at least on the personal level. In particular, examples of mind-wandering and other cases of association do not constitute mental projects. They do not show the required unity partly because they lack an overall representational purpose, and therefore also a narrative structure. The other reason for the absence of personal-level unity is that instances of mind-wandering do not possess any other feature which could be used to explain why they contain exactly these experiences and thoughts and no other mental episodes.

The associative links between the successive mental episodes are insufficient to establish the unity of the resulting sequences. In particular, there is no answer to the question of when or where one instance of mind-wandering stops and another starts. For instance, how many associative sequences of experiences and thoughts are involved in James' or Hobbes' example? Why should we take the set of mental representations concerned with the clock and the watchmaker to be part of the same unified mental phenomenon as, say, the set of mental representations concerned with the value of gold and of the dollar? It might be proposed that we just have to look at when associative causation (i.e. some specific form of causation in virtue of representational content) actually starts and stops in order to delineate distinct sequences of mind-wandering. But this proposal would not work because the identity and unity of mental phenomena is partly a matter of what happens in counterfactual conditions. For example, we need answers to questions like whether the associative sequence would still be the same if it would link up more mental episodes, or different ones - that is, questions concerning the essentiality of certain actual or potential episodic constituents. In the case of focused daydreams, we can answer such questions by reference to their unifying representational purpose, as well as to their resulting narrative structure.

Instances of mind-wandering are also not unified by the non-representational purposes for which we might be engaged in them. It is sometimes our choice to let our mind wander off freely (or, if one prefers, to abstain from mentally acting). We may 
choose to do this, say, for its own sake, or because we want to relax ourselves, or because we take pleasure in being surprised by whatever sequence of mental episodes unfolds in our mind. But none of these or similar goals can unify the resulting sequences of mental episodes. First of all, the end of letting our mind wander off is completely unspecific and does not distinguish between the various instances of mindwandering that involve it. Accordingly, it does not help to determine which mental episodes form part of which purposive instances of mind-wandering. The other, secondary goals also leave it completely open which mental episodes are able or required to achieve them. Of course, we could use our knowledge about which experiences and thoughts are likely to further our end of unwinding, say, and act on our respective intention by producing in us these mental episodes. But if we act in this way, we end up engaging in a focused daydream, rather than letting our mind wander off.

Other features of the mental episodes involved in mind-wandering - such as their temporal or causal relations, or their concern with the same entities - are equally unable to unify the episodes in a distinct mental phenomenon. Many mental episodes are temporally or causally linked to each other in the same way as the episodes involved in mind-wandering, without thereby together forming a self-contained segment within the stream of consciousness. So, the existence of such relations is not sufficient for unity. Referential sameness, on the other hand, often fails to be present in cases of associative mind-wandering. After all, the point of association is to switch attention from one thing to something completely different. Besides, even if the episodes in the associative sequence happen to represent the same entities, they do so only arbitrarily. It is always possible that an associative chain, that has so far been representationally homogenous, continues with mental representations of very different events, persons or objects. And there is no answer to the question of whether such switches in subject matter also lead to changes in the identity of the instance of mind-wandering concerned. Hence, referential sameness cannot be used to unify occurrences of mind-wandering, even if it may be responsible for the unity of other segments of the stream of consciousness, such as temporally extended (and possibly interrupted) aesthetic experiences of particular artworks (Dorsch 2000, ch. 1).

\section{Conclusion}

Focused daydreams are instances of imaginative mental agency: they are mental projects with the purpose of voluntarily producing specific mental representations. Their purposiveness provides them with unity and a narrative structure. Instances of mind-wandering, on the other hand, do not constitute mental projects and, in particular, lack any overall representational purpose and narrative structure. Indeed, mindwandering does not even give rise, on the personal level, to self-contained sequences of mental episodes. Rather, it just consists in a succession of loosely connected mental episodes and cannot be distinguished from other segments of the stream of consciousness in a non-arbitrary manner. In particular, there is no answer to the question of which mental episodes or which associative steps between them are essential to any given associative chain. 
This does not mean, however, that mind-wandering cannot involve mental or even imaginative agency. For the associatively linked mental episodes may be-and often are-simple or complex instances of such agency. This is perhaps also the main reason why we tend to wrongly take focused daydreaming and mind-wandering to be instances of one and the same mental phenomenon (i.e. 'daydreaming'): they both typically involve imaginative agency, albeit in very different ways - either because such agency is constitutive of them (focused daydreaming), or because it is constitutive of some of their episodic elements (mind-wandering). ${ }^{10}$ In other words, instances of mind-wandering are closely connected to focused daydreams - and thus also to imaginative agency-because they usually involve such imaginative mental projects as elements in their associative chains. ${ }^{11}$

Acknowledgments Research on this work was funded by the Swiss National Science Foundation.

\section{References}

Buckner, R.L., J.R. Andrews-Hanna, and D.L. Schacter. 2008. The brain's default network-Anatomy, function, and relevance to disease. Annals of the New York Academy of Sciences 1124: 1-38.

Budd, M. 1989. Wittgenstein's philosophy of psychology. London: Routledge.

Carroll, N. 2001. On the narrative connection. In Beyond aesthetics, ed. Carroll, 118-133. Cambridge: Cambridge University Press.

Davidson, D. 1980. Actions and events. Oxford: Clarendon.

Dorsch, F. 2000. The nature of aesthetic experiences. London: UCL Discovery.

Dorsch, F. 2010. The unity of hallucinations. Phenomenology and the Cognitive Sciences 9: 171-191.

Dorsch, F. 2012. The unity of imagining. Berlin: De Gruyter.

Dorsch F. 2015. Imagination. London: Routledge.

Giambra, L.M. 1995. A laboratory based method for investigating influences on switching attention to task unrelated imagery and thought. Consciousness and Cognition 4: 1-21.

Goldie, P. 2003a. Narrative and perspective: Values and appropriate emotions. In Royal institute of philosophy supplements series, ed. A. Hatzimoysis, 201-220. Cambridge: Cambridge University Press.

Goldie, P. 2003b. Narrative, emotion and perspective. In Imaginative and the arts, ed. M. Kieran and D. Lopes. London: Routledge.

Hobbes, T. (1651/1996). Leviathan. Cambridge: University Press.

Ichikawa, J. 2009. Dreaming and imagination. Mind and Language 24: 103-121.

James, W. 1890/1981. The principles of psychology. Cambridge: Harvard University Press.

Lamarque, P. 2004. On not expecting too much from narrative. Mind and Language 19(4): 393-408.

McGinn, C. 2004. Mindsight. Cambridge: The MIT Press.

McMillan, Rebecca L., S.B. Kaufman, and J.L. Singer. 2013. Ode to positive constructive daydreaming. Frontiers in Psychology 4: 626.

\footnotetext{
${ }^{10}$ Another explanation of why we might be tempted to treat the two phenomena as very similar, or even as differing at best in degree, is that they both involve a shift in attention away from the external world and our interaction with it. In particular, both focused daydreaming and mind-wandering are typically unconcerned with our actual situation or tasks. This similarity might also be further elucidated by the - not uncontroversial -idea that both phenomena centrally involve the brain's 'default network' (see Buckner et al. 2008, especially section III, and McVay and Kane, 2010, for discussion).

${ }^{11}$ I would like to thank Davor Bodrozic, Malcolm Budd, Magnus Frei, David Harris, Sebastian Gardner, Peter Goldie, Rob Hopkins, Mike Martin, Lucy O'Brien, Thomas Pink, Tobias Schlicht, the editors and the anonymous referees for their stimulating discussions and/or very helpful comments on previous drafts. My work on this article was funded by the Swiss National Science Foundation, as part of the research project The Normative Mind (PP00P1_139004).
} 
McVay, J.C., and M.J. Kane. 2010. Does mind wandering reflect executive function or executive failure? Psychological Bulletin 136(2): 188-207.

Metzinger, T. 2013. Why are dreams interesting for philosophers? The example of minimal phenomenal selfhood, plus an agenda for future research. Frontiers in Psychology 4: 746.

O'Shaughnessy, B. 2000. Consciousness and the world. Oxford: Oxford University Press.

Peacocke, C. 1985. Imagination, experience and possibility. In Essays on Berkeley, ed. J. Foster and H. Robinson. Oxford: Clarendon.

Pink, T. 1996. The psychology of freedom. Cambridge: Cambridge University Press.

Pink, T. 2003. Action and self-determination. Intellectica 36(37): 247-259.

Regis, M. 2013. Daydreams and the function of fantasy. London: Palgrave Macmillan.

Singer, J.L., and V.G. McCraven. 1961. Some characteristics of adult daydreaming. Journal of Psychology 51: 151-164.

Schooler, J.W., J. Smallwood, K. Christoff, T.C. Handy, E.D. Reichle, and M.A. Sayette. 2011. Metaawareness, perceptual decoupling and the wandering mind. Trends in Cognitive Sciences 15(7): 319-326.

Smallwood, J., and J.W. Schooler. 2006. The restless mind. Psychological Bulletin 132: 946-958.

Smallwood, J. 2013. Distinguishing how from why the mind wanders: a process-occurrence framework for self-generated mental activity. Psychological Bulletin 139: 519-535.

Sutton, J. 2010. Carelessness and inattention: mind-wandering and the physiology of fantasy from Locke to Hume. In The body as object and instrument of knowledge. Embodied empiricism in early modern science, ed. C. Wolfe and O. Gal. Dordrecht: Springer.

Wegner, D.M. 1997. Why the mind wanders. In Scientific approaches to consciousness, ed. J.D. Cohen and J.W. Schooler, 295-315. Mahwah: Erlbaum.

Williams, B. 2002. Truth and truthfulness. Princeton: University Press.

Wollheim, R. 1973. Imagination and identification. In On art and the mind, ed. R. Wollheim. Cambridge: Harvard University Press.

Wollheim, R. 1984. The thread of life. New Haven: Yale University Press.

Zangwill, N. 2006. Daydreams and anarchy. Philosophy and Phenomenological Research 73(2): 253-289. 